\title{
CUSTOM-MADE RHEOMETER FOR THE EXPERIMENTAL STUDY OF POLYURETHANE RESIN PU9010
}

\author{
Luciano Fissore*, Stefan Diebels \\ Saarland University, Institute of Applied Mechanics, Campus A4.2, 66123 Saarbrücken, Germany \\ * corresponding author: s8lufiss@stud.uni-saarland.de
}

\begin{abstract}
Polyurethane resins are well-known examples in aerospace industry, electronics and automotive as an adhesive or coating because of their broad spectrum of properties. They are obtained by a process called curing, where in the initial uncured state the mixture of monomers exhibits a viscous behavior and at the end of the polymerization reaction, a solid with viscoelastic properties is formed. The shrinkage due to cross-linking and thermal expansion cause the generation of residual stresses which often result in broken adhesive layers. Therefore, the reduction of such problems is of utmost importance. In this study, a rheometer capable to conduct oscillatory-strain-controlled experiments was built in order to apply large deformations not only to the sample at the beginning of the reaction but also during the curing process and to the fully cured adhesive.
\end{abstract}

KEYWORDS: Rheology, curing, dynamics.

\section{INTRODUCTION}

The studies on polymers have increased at a high rate in the past few years not only due to their broad spectrum of properties but also because of its high versatility of production. Among them are cross-linked adhesives, which are a special kind of polymer because they undergo a chemical reaction that transforms the liquid sample into solid called curing. At the beginning, the uncured polymer solution behaves as a deformable viscous liquid which can only withstand hydrostatic loads. During curing, the polymer chains cross-link to each other forming a 3D network which means that there is an increase in its viscosity, in its stiffness and in relaxation or creep effects. The cured and solidified polymers are used in electronics and automotive parts, among others.

There are researchers, like [1, that study the chemical reaction; others study the way the chemical reaction affects how the resin behaves mechanically, chemically or thermally [24]. Moreover, during the curing process a shrinkage up to a volumetric strain of $10 \%$ can be observed, leading to large residual stresses. Depending on the mechanical properties this inhomogeneous stress distribution may damage the adhensive layer even without external loading [5]. There are also investigations that try to find suitable mathematical equations to model the curing reaction as well as material models to simulate the time-dependent mechanical behavior during and after curing [6, 7].

One way of studying how the mechanical properties change with time is by using a rheometer. The aim of this study is to build a rheometer capable of applying large deformations to the sample not only in liquid state but also in solid state. Due to the fact that commercial rheometers are normally used to study the rheology of liquids, the torque needed in this case is 10 times lower than the maximum torque that this rheometer should apply for the fully cured solid. Furthermore, once the rheometer is built, it will be used to study how the deformation, the frequency and temperature influence the properties of the fully cured resin.

\section{EXPERIMENTAL}

\subsection{MATERIALS}

The reactants to obtain Polyurethane PU9010 are: Desmodur VP.PU 1806 (Isocyanate), Baygal K55 (Triol) with $M_{\mathrm{n}}=444 \mathrm{~g} / \mathrm{mol}$, Desmophen $3600 \mathrm{Z}$ (Diol) with $M_{\mathrm{n}}=2000 \mathrm{~g} / \mathrm{mol}$. Baygal K55 is the crosslinking agent because of its 3 functional hydroxils. The reaction is very sensitive to the presence of water. Hence, the experimentall test must be perfomed in a humidity-controlled environment [8].

\subsection{Rheological EXPERIMENTAL Plan}

Dynamic measurements in oscillatory mode are planned with strain amplitudes from $2 \%$ to $100 \%$. The tests will be performed during the reaction as well as once it is finished. The aim is to observe if there is a possible coupling between the curing reaction and the strain amplitude and how the mechanical properties are influenced by the curing and loading history. Furthermore, each oscillatory test will be performed at different temperatures and frequencies, from $T=25^{\circ} \mathrm{C}$ to $T=70^{\circ} \mathrm{C}$ and from $1 \mathrm{~Hz}$ to $15 \mathrm{~Hz}$, respectivetly.

\section{RHEOMETER}

The design of the rheometer and the mechanism to put it in motion is shown in Figure 1 . 


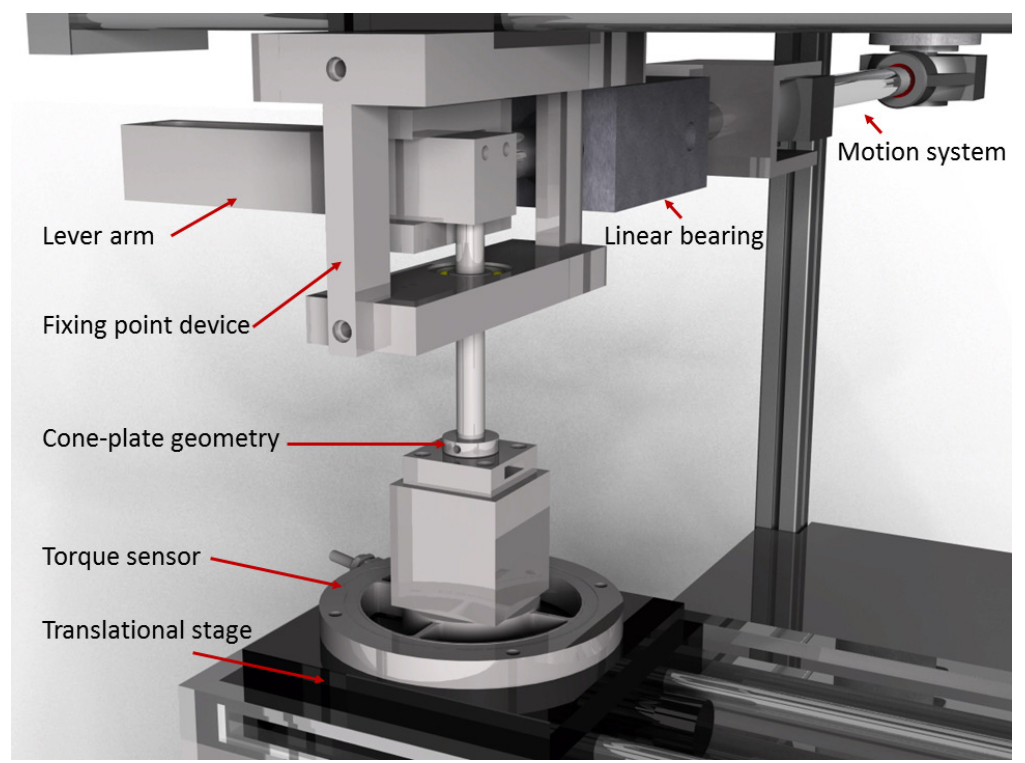

FIGURE 1. Rheometer and motion mechanism drawn in Catia V5 ${ }^{\circledR}$.

\subsection{Geometry}

The most important part of a rheometer is the implemented geometry because it determines not only how the sample is deformed during the process but also it determines the torque needed to apply large deformations to the sample. In this case, to examine the curing reaction of a resin without fillers or nanoparticles, a plate-plate or a cone-plate-geometry could be used. The main difference between them, and also the main reason to exclude the plate-plate geometry, is that with the former geometry the applied strain and strain rate do not depend on the radius of the sample. In Table 1 and in Figure 2 the dimensions are shown. Given the fact that the tip of the cone could be in contact with the plate, it was decided to use a truncated cone forming a gap of $50 \mu \mathrm{m}$ in order to avoid possible wear on the lower plate and spurius tensions in the measurement.

\begin{tabular}{llc}
\hline 1. & Radius & $10 \mathrm{~mm}$ \\
2. & Angle & $2^{\circ}$ \\
3. & Gap & $50 \mu \mathrm{m}$ \\
\hline
\end{tabular}

TABle 1. Characteristic features of the cone-plate geometry.

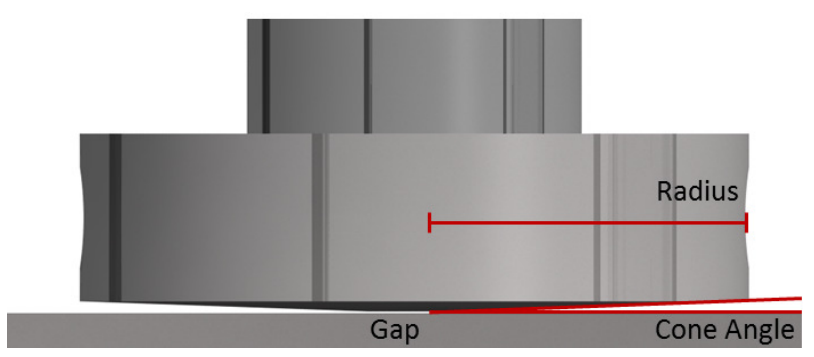

FiguRE 2. Implemented cone-plate geometry.
Because of the nature of the chemical reaction, once it finishes, both the cone and the upper part of the plate must be discarted.

\subsection{Motion}

Based on the fact that a oscillatory motion is needed, a crank-slider mechanism was selected because it can transform the pure rotational motion of a normal motor into a planar translational oscillatory motion. The amplitude of oscillation is equal to the eccentric position of the main connecting rod related to the rotational axis of the motor. The problem is that this eccentricity, R, also induces dynamic forces or vibrations to the machine. In Figure 3 a sketch of the mechanism is showed where $\mathrm{R}$ is the eccentricity, $\mathrm{L}$ is the length of the main connecting rod and $\mathrm{v}$ is the velocity of the slider.

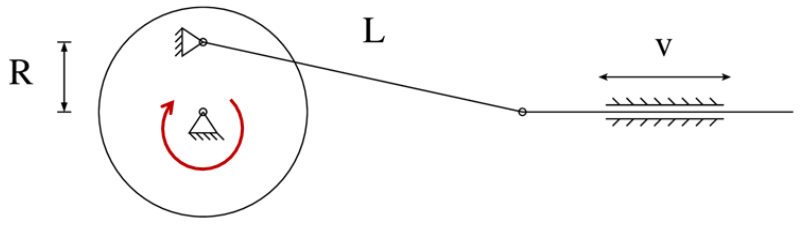

FiguRE 3. Crank-Slider mechanism.

\subsubsection{DynAmic ForCES}

The reduction of dynamic forces is of best interest because they affect the absolute position of the cone in relation to the fixed plate and therefore affect the final measurements. As it can be seen in Equation 1 .

$$
F_{\mathrm{D}} \approx m R w^{2}\left(\cos (w t)+\lambda \cos (2 w t)-\frac{\cos (4 w t) \lambda^{3}}{4}\right),
$$

the dynamic force, $F_{D}$, is aproximated to the 4 th harmonic and depend on the mass $\mathrm{m}$, the eccentricity $\mathrm{R}$ and $\lambda=\frac{L}{R}$, which is the relation between the 
length of the main connecting rod and the eccentricity. Therefore, one way of reducing the dynamic forces is by reducing the eccentricity as well. However, this means that the strain amplitude also decreases. where $\omega$ is the frequency of oscillation and t is time.

\subsubsection{LEVER ARM}

The planar translational oscillatory motion is transformed into a rotational oscillatory motion using a lever arm (Figure 4 because, in principe, this would give the opportunity to apply high torque using small forces. Furthermore, if it is possible to change the length of the lever arm, the mentioned reduction in strain amplitude could be balanced based on the similarity of triangles. This means that a constant deformation or, in other words, a constant angle of rotation can be obtained if the tangent of the angle of rotation is kept constant as well.

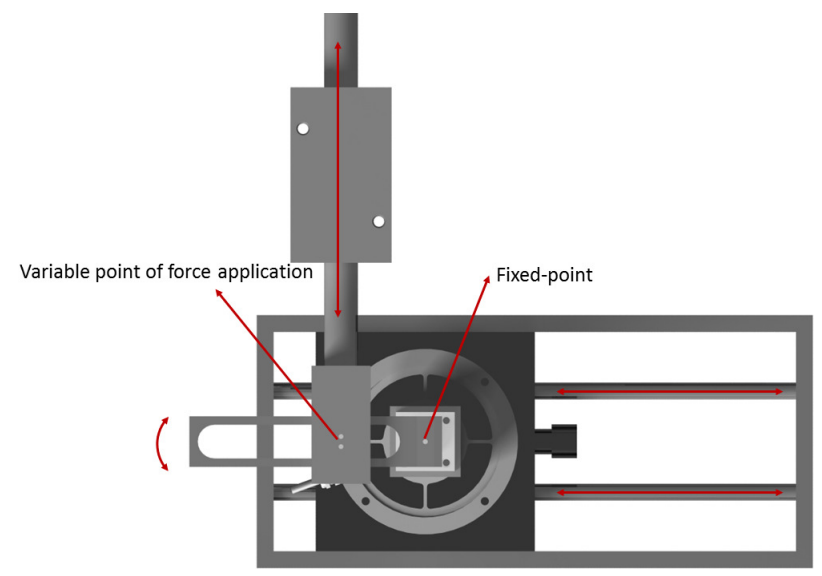

FiguRE 4. Lever arm mechanism implementation.

\subsection{SEnSORS}

\subsubsection{TEMPERATURE}

The temperature measurement range is accomplished by a ceramic heating plate from Misumi ${ }^{\circledR}$, beneath the plate in Figure 1. This kind of device are small enough to fit in the rectangular hole that can be seen in Figure 2 and its maximum operating temperature and power are high enough for this study.

In order to be able to measure the temperature directly on the sample, the temperature sensor is placed near the bottom part of the truncated cone in Figure 1). Keeping in mind the dimensions of the cone and that it has to be changed after each test, the sensor must be small and cheap in order to be able to change it once each test is over. Therefore, a NTC thermoresistor was selected since its high temperature reaction rate and its small size.

\subsubsection{DEFORMATION}

As there are many parts in motion and many places where forces are transmitted from body to body, it is important to measure the deformation directly on the sample. The strain amplitude, $\gamma$, can be transform into degrees of rotation with the help of Table 1 and Equation 2,

$$
\gamma=\frac{\theta}{\tan \left(2^{\circ}\right)},
$$

where $\theta$ is the angle of rotation. Applied to the upper and lower limit of the measurement range, the strain amplitude limits are

(1.) The corresponding angle of rotation for a

$$
\gamma_{\text {lower }}=2 \% \text { is } \approx 0.046^{\circ} \text {. }
$$

(2.) The corresponding angle of rotation for a

$$
\gamma_{\text {upper }}=100 \% \text { is } \approx 2^{\circ} \text {. }
$$

The aim is to measure the deformation in during the entire oscillatory motion, therefore, the sensor must have not only a fast reaction to changes but also very high resolution. A sketcht of the deformation measurement is shown in Figure 5. The idea is to placed a laser on the oscillating surface of the axis of the cone and a photodiode sensor C10443-01 from Hammamatsu ${ }^{\circledR}$ in a distance $\mathrm{x}$ mm. These sensors can measure how an incident laser beam moves along its surface with a resolution of $0.5 \mu \mathrm{m}$ giving the chance to measure the $0.046^{\circ}$ of oscillation as the travel distance of the laser beam.

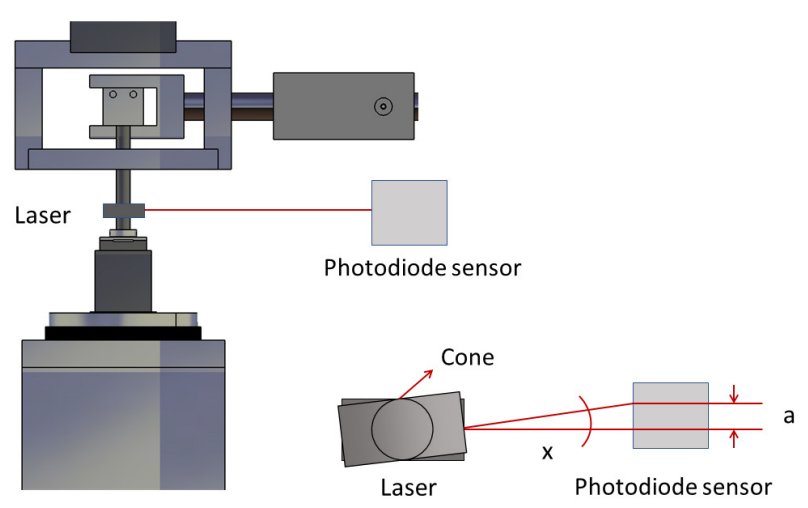

FIGURE 5. Deformation measurement by a laser and a photodiode array.

\subsubsection{TORQUE}

In this rheometer the torque sensor that was chosen is a reaction torque sensor because it fits the design better than dynamic torque sensors. However, as the sample at early stages of the reaction is a liquid and later on it transforms into a solid, two sensors with different resolutions must be placed in the device. The idea is to build an adapter which allows both sensors to be placed at the same time and when a maximum value of torque is reached, the bigger sensor is activated avoiding possible damage on the smaller one.

Given the fact that the resolution of the sensor depends on the full scale value, the big sensor, on the one hand, must be selected according to the stiffness of the fully cured sample. On the other hand, 


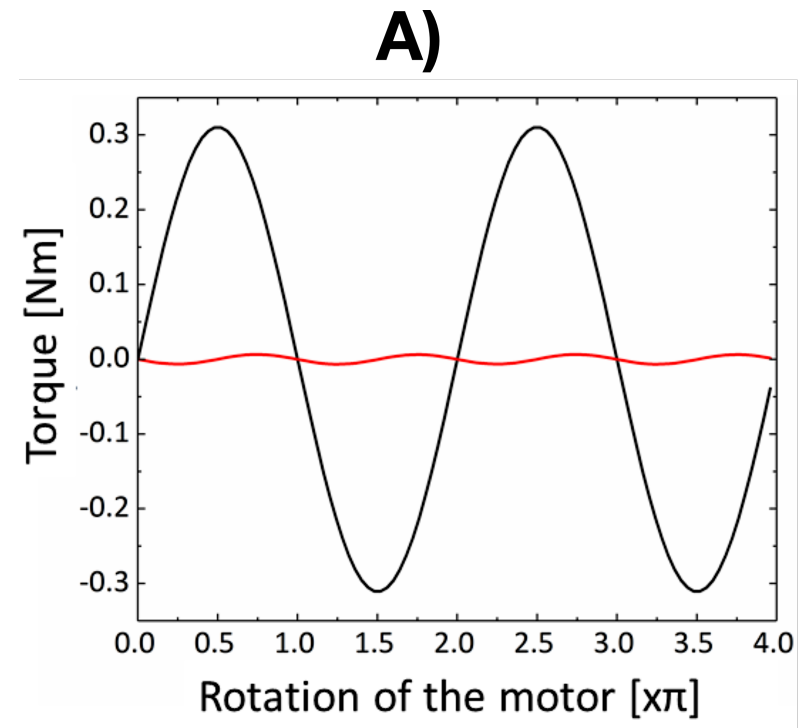

_ Applied torque on the sample

Applied inertial torque on the sample

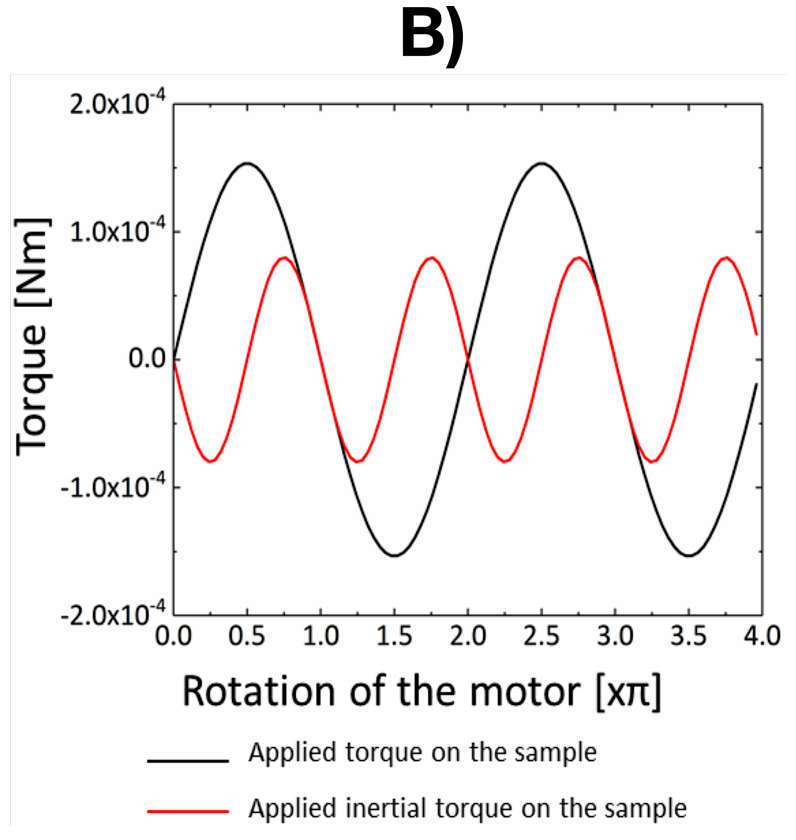

Figure 6. Dynamic and applied torque components for: (A) lever length $25 \mathrm{~mm}$ and eccentricity $0.9 \mathrm{~mm}$; (B) lever length $125 \mathrm{~mm}$ and eccentricity $0.1 \mathrm{~mm}$

the measurement range of the small sensor should include both the lowest value that the other sensor can measure and the minimum value for this kind of polymer during the liquid phase. Therefore, a $20 \mathrm{Nm}$ reaction torque sensor from ME-Mesysteme $\mathrm{GmbH}^{\circledR}$ with a resolution of $0.1 \%$ fs and also a $0.3 \mathrm{Nm}$ with a resolution of $0.1 \%$ fs as well were selected.

\section{Results}

The needed torque to deform the fully cured sample up to $100 \%$ is calculated using the radius of the cone $\mathrm{R}$, showed in Table 1 and the shear modulus $\mathrm{G}$, of the fully cured PU9010 8.

$$
\begin{gathered}
\tau=G \gamma, \\
M=\tau \frac{2}{3} \pi R^{3} .
\end{gathered}
$$

First the maximum shear stress $\tau$, was calculated using Equation 3 and then substituted into Equation 4 to obtain $\approx 8.4 \mathrm{Nm}$ of torque.

Furthermore, the possibility of changing the length of the lever arm gives good results on keeping low the dynamic forces. The applied torque and the dynamic torque on the sample for minimum and maximum values of deformation were obtained. In 6 can be observed the change of both torques as a function of the motor degree of rotation. When the sample is deformed $100 \%$, an eccentricity of $R=0.9 \mathrm{~mm}$ and lever arm of $25 \mathrm{~mm}$ are needed(A), while in (B) when the sample is deformed $2 \%$ the used eccentricity is $R=0.1 \mathrm{~mm}$ and the used lever arm is equal to $125 \mathrm{~mm}$. Moreover, deformations in between these limits will be obtained by finding the best combination of both variables. In consequence, keeping a small eccentricity constant, a certain deformation can be applied by changing only the position of the oscillating connecting rod in relation to the fixed point (Figure (4).

As stated earlier, one of the reasons for which a commercial rheometer can not be used in this kind of study was the lack of high-torque and high-frequency features. Therefore, given the final design of the device, it can also be seen in Figure 6 that the torque that the motor has to be able to apply is more than 20 times lower than the needed torque calculated previously. As a consequence, it is possible to find a suitable motor capable of suppling a torque of $0.3 \mathrm{Nm}$ at $15 \mathrm{~Hz}$. For instance, the MDrive 34 plus from Schneider Electric ${ }^{\circledR}$ is an appropiate motor because it has a Torque/Frequency ratio suitable for this application.

\section{Conclusions}

In the present work, a rheometer was succesfully design as far as mathematical calculations can predict. Thereby, different rheological test can be performed changing the maximum deformation, the temperature and the frequency. Furthermore, the torque that the motor has to apply could be reduced using CrankSlider mechanism and a lever arm making it possible to find a suitable motor.

The final design can be seen in Figure 7 in which also a glovebox is included for a controlled atmosphere. This leads to future work, in which the next step would be to design the system to control both the humidity and temperature inside the glovebox and also to generate the interface for the control of the device in LabView ${ }^{\circledR}$ a software from National Instruments ${ }^{\circledR}$. 


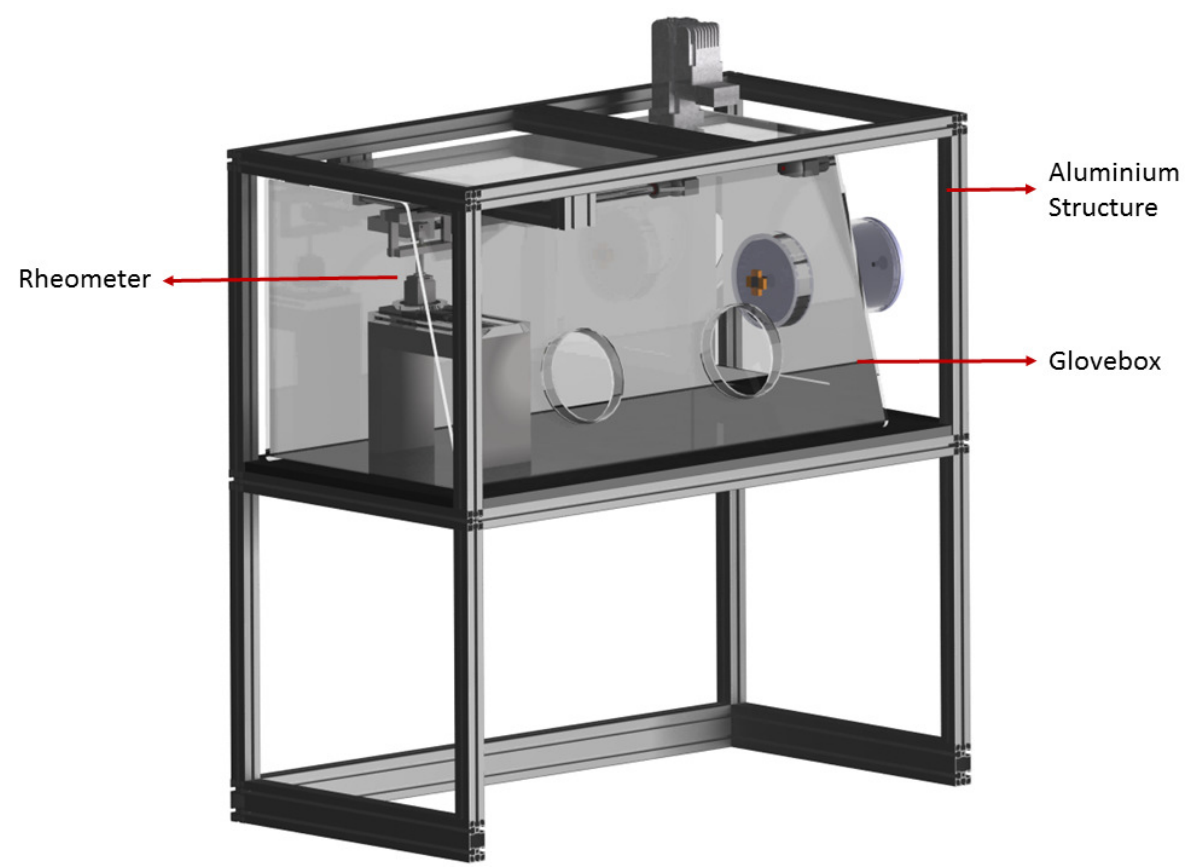

Figure 7. Final design of a MDA machine for rheometric analysis of PU9010.

\section{ACKNOWLEDGEMENTS}

The research was supported by DAAD and directed by the Chair of Applied Mechanics in Saarland University. Both finantial support and guidence during the reasearch is gratefully recognized.

\section{REFERENCES}

[1] S. Montserrat, J. Malek. A kinetic analysis of the curing reaction of an epoxy resin. Thermochimica Acta 228:47-60, 1993. DOI:10.1016/0040-6031(93)80273-D

[2] S. Saseendran. Effect of degree of cure on viscoelastic behavior of polymers. Master's thesis, Luleå University of Technology, 2016.

[3] N. Illie, A. Simon. Effect of curing mode on the micro-mechanical properties of dual-cured self-adhesive resin cements. Clinical Oral Investigations 16:505 - 512, 2009. DOI:10.1007/s00784-011-0527-x.

[4] M. G.-M. E. Papadopoulos, S. Clarke. A thermal and rheological investigation during the complex cure of a two-component thermoset polyurethane. Journal of Applied PolymerScience 114:3802-3810, 2009. DOI:10.1002/app.30987
[5] M. H. A.J. Hudson, S.C. Martin, J. Spelt. Optical measurements of shrinkage in uv-cured adhesives. ASME 124:352-354, 2002.

[6] P. S. M. Hossain. Modelling and simulation of the curing process of polymers a modified formulation of the arruda-boyce model. Archives of Mechanics 63:621-633, 2011. DOI:10.1002/pamm.201110186

[7] B. Y. C. Liebl, M. Johlitz, A. Lion. Three-dimensional chemo-thermomechanically coupled simulation of curing of adhesives including viscoplasticity and chemical shrinkage. Computational Mechanics 49:603-615, 2011. DOI:10.1007/s00466-011-0663-9.

[8] M. B. W. Possart. Adhesive Joints: Ageing and Durability of Epoxies and Polyurethanes. Wiley-Book, 2018. 